\title{
An Interpenetrating Network Composite for a Regenerative Spinal Disc Application
}

\author{
A.H. Chan ${ }^{\mathrm{a}, *}$, P.C. Boughton ${ }^{\mathrm{a}}$, A.J. Ruys ${ }^{\mathrm{a}}$, M.L. Oyen ${ }^{\mathrm{b}}$ \\ ${ }^{a}$ Biomedical Engineering, Engineering \& IT, University of Sydney, Australia \\ ${ }^{b}$ Cambridge University Engineering Department, Cambridge University, UK
}

\begin{abstract}
Severe degeneration of the intervertebral disc has an immensely debilitating effect on quality of life that has become a serious health and economic burden throughout the world. The disc plays an integral role in biomechanical movement and support within the spine. The emergence of tissue engineering endeavours to restore the structural characteristics and functionality of the native tissue. Hydrogels have been widely investigated as a candidate for regeneration of the gelatinous nucleus pulposus due to its architectural resemblance and fluid retention characteristics. However, hydrogels are often limited due to small compressive stiffness and tear resistance, leading to extrusion complications. Reinforcement of the hydrogel network using polymeric scaffolds may address these issues of inadequate mechanical properties and implant instability. This study investigates the potential of a carrageenen gel-infused polycaprolactone scaffold for nucleus pulposus tissue engineering. Mechanical properties were characterised using viscoelastic and poroelastic frameworks via microindentation. The incorporation of polymeric reinforcement within the gels increased material stiffness to that comparable to the native nucleus pulposus, however permeability was significantly greater than native values. A preliminary cell evaluation culturing NIH 3T3s over 21 days suggested the incorporation of polymeric networks also enhanced cellular proliferation compared to gels alone.
\end{abstract}

Keywords: hydrogel, nucleus pulposus, tissue engineering, intervertebral disc, carrageenan, PCL

\section{Introduction}

Lower back pain is a growing health and economic burden in society, costing the US over $\$ 100$ billion per annum in direct and indirect costs [15]. Chronic back pain is often attributed to intervertebral disc abnormalities derived from

\footnotetext{
*Corresponding author

Email address: annabelle.chan@sydney.edu.au (A.H. Chan)

$U R L:$ http://oyenlab.org (M.L. Oyen)
} 
trauma, ageing processes, metabolic disorders or degenerative disc diseases [33]. Cases where neurological pain is debilitating and conservative treatments are insufficient, surgical intervention is required. Techniques involving decompression of the spine and fusion implants typically yield good short term clinical results with pain alleviation, however do not restore physiologic mobility.

Tissue engineering has opened a paradigm for intervertebral disc regeneration and the restoration of natural joint biomechanics. As intervertebral disc degeneration is often believed to originate from the central gelatinous component, the nucleus pulposus, hydrogels have been widely investigated as a potential replacement due to its structural and viscoelastic similarities. The gelatinous materials exhibit similar water retention and attraction properties as well as the characteristic long-range network order similar to the nucleus.

While gels resemble the natural nucleus pulposus on one level of molecular order, they lack the heirarchical structure and mechanical stability integral for good connectivity and cohesion with hard tissues. This is inevitably problematic in vivo due to leakages of biomolecules fundamental for localised regeneration and the inability to resist collapse in the spinal joint [40, 2, 38]. As a result, implants harnessing hydrogels place significant restrictions on implant performance and have known incidences of expulsion [20, 21, 7, 17, 12].

Hydrogel-polymer composites have been explored, harnessing the structural and mechanical elements of both materials for nucleus pulposus regeneration $[6,16]$. Studies have focused on the integration of hydrogels with electrospun fibres to replicate the native extracellular matrix [38, 37]. It was shown that the inclusion of nanofibers in hydrogels induced varying levels of reinforcement depending on fabrication methods. In addition, it was hypothesized that further reinforcement could be achieved by altering fibre alignment and gel concentrations [38].

Carrageenan is a naturally occurring anionic sulphated polysaccharide extruded from red seaweed of the Rhodophyceae family [29]. Carrageenan has been investigated in biomedical applications for cell encapsulation and drug delivery [5, 27, 28]. Types of carrageenan are determined by the number of sulphated groups with kappa $(\kappa)$, iota $(l)$ and lambda $(\lambda)$ possessing one, two and three sulphated groups per dissacharide unit respectively [24]. Advantages of carrageenan over other hydrogels include its thermoreversible and ionic hydrogel characteristics which enable mild conditions for cell encapsulation and versatility in manufacturing and processing [28].

$\kappa$-carrageenan has the potential for tissue engineering applications due to its gelation properties, mechanical strength and its resemblance to natural glucosaminoglycans [24]. In vivo screenings of an electrospun polycaprolactone (PCL)-carrageenan composite indicated that cells readily adhered to the scaffold [1]. In addition, carrageenan exhibits thixotropic characteristics which resemble nucleus behaviour as it can become fluidic when experiencing pressure, acting both as a fluid and a viscoelastic solid [24].

While carrageenan exhibits desirable mechanical and material similarities to the nucleus pulposus, it has not been considered for intervertebral disc regeneration. The present study evaluates the suitability of $\kappa$-carrageenan for the 
augmentation of a polymer scaffold to replicate connective tissue characteristics, with the gel functioning as a temporal incompressible phase in a nucleotomized spinal disc. Particular focus is placed on a regenerative non-injectable solution as a component of a total disc replacement, rather than an injectable hydrogel composite treatment, to provide a more continuous interface between the central nucleus and annulus fibrosus.

\section{Methods}

\subsection{Sample Preparation}

Scaffold precursors were formed using sucrose crystals infused in a mixture of 4:1 acetone and PCL $\left(\mathrm{M}_{\mathrm{n}}=80,000 \mathrm{~g} / \mathrm{mol}\right.$; Perstrop Capa ${ }^{\mathrm{TM}}$ 6800, Australia) heated at $50{ }^{\circ} \mathrm{C}$ over night. Precursors were processed using particle leaching techniques and allowed to dry at room temperature. Polymer scaffolds were made to approximate dimensions of $18 \mathrm{~mm} \times 17 \mathrm{~mm} \times 11.5 \mathrm{~mm}$ and had an average porosity of $93.7 \pm 0.2 \%$.

Carrageenan gels (PT Forisa Nusapersada, Indonesia) were made to concentrations of $1 \%, 2 \%$ and $3 \%$ by stirring at over $85^{\circ} \mathrm{C}$ for 5 minutes. Gel solutions were allowed to rest for one minute to allow air bubbles to escape and subside. Pure gels were made into varying geometries depending on test type by casting in moulds with a $40 \mathrm{~mm}$ diameter and $5 \mathrm{~mm}$ height, then cut to $5 \mathrm{~mm}$ and $10 \mathrm{~mm}$ diameters using a hole punch tool after allowing to set overnight .

Gel infusion within interpenetrating network (IPN) scaffolds was performed using a custom syringe system at $50^{\circ} \mathrm{C}$. Microscopy was used to verify infusion efficacy and consistency. The overall experimental setup is outlined in Figure 1.

\subsection{Mechanical Testing}

The mechanical properties of the gels and IPN scaffolds were characterised using microindentation. Indentation was carried out using an Instron 5544 universal testing frame (Canton, MA, USA) with a $5 \mathrm{~N}$ load cell. A spherical indenter tip of radius $(R) 8 \mathrm{~mm}$ and $3 \mathrm{~mm}$ was used on gels and IPN scaffolds respectively to induce a maximum displacement $\left(h_{\max }\right)$ of $0.5 \mathrm{~mm}$ to conform to $5 \%$ strain as outlined in $\varepsilon=0.2 \sqrt{\frac{h_{\max }}{R}}$ [14]. A small preload of $0.01 \mathrm{~N}$ was applied prior to a 10 second ramp to peak displacement. Viscoelastic responses were then examined over 300 seconds.

Gel samples of $40 \mathrm{~mm}$ diameter and $5 \mathrm{~mm}$ thickness and were secured to the plate using acrylonitrile and immersed in PBS. Prior to testing, infused gels were cut in half to a thickness of approximately $5.5 \mathrm{~mm}$ to ensure a flush gel-scaffold surface. 3-5 tests were carried out on 4 samples of each gel concentration and gel-infused scaffold. Viscoelastic indentation analyses were performed using an elastic-viscoelastic approach previously validated via numerical integration and finite element analysis $[22,36,30]$. 


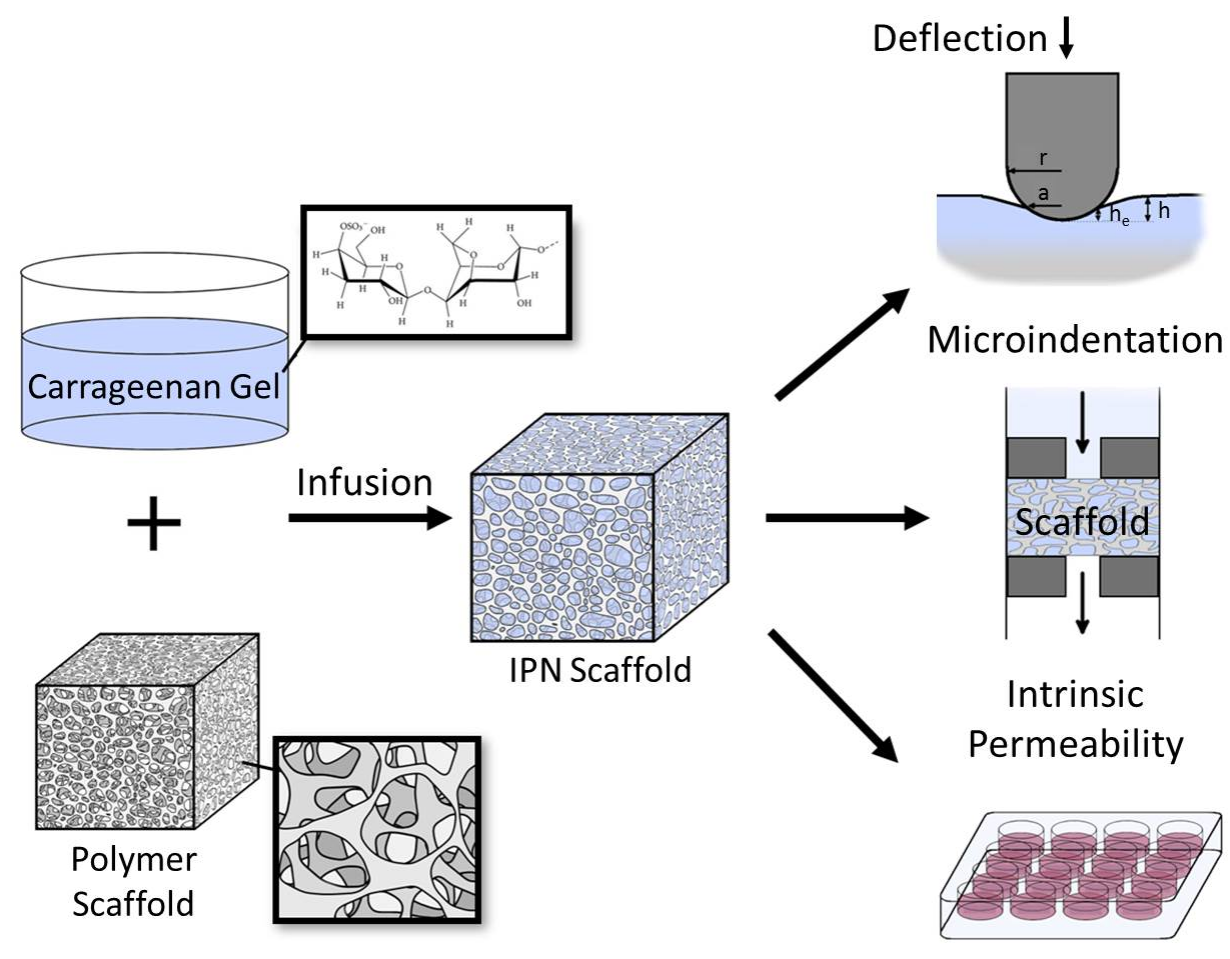

Cell Biocompatibility

Figure 1: A schematic illustration of the experimental setup investigating carrageenan gelpolymer composites. Interpenetrating networks were manufactured by infusing gels into scaffolds then characterised mechanically and biologically by means of microindentation, permeability and cell viability.

Succinctly, time dependent mechanical properties were analysed via the principle of elastic-viscoelastic correspondence using a Bolzmann hereditary integral formulation based on an elastic Hertzian contact solution [22]. As seen in Equation 1, indentation load $P(t)$ was related to indentation displacement $h(t)$ and time $t$, assuming a constant Poisson's ratio $v$ of 0.5 .

$$
P(t)=\frac{8 \sqrt{R}}{3(1-\nu)} \int_{0}^{t} G(t-u)\left[\frac{d}{d u} h^{\frac{3}{2}}(u)\right] \mathrm{d} u .
$$

The function at which stress relaxes over time due to viscous effects in the material was assumed to take the form of a Prony series as expressed in Equation 2. From this, the calculated parameters included the instantaneous modulus $\left[G_{0}=2 G(0)(1+v)\right]$, equilibrium modulus $\left[G_{\infty}=2 G(\infty)(1+v)\right]$, and the viscoelastic ratio $f\left(f=\frac{G_{\infty}}{G_{o}}\right)$, a measure of viscoelastic deformation. Viscoelastic ratios indicated the level of elasticity and viscocity exhibited by the material, 
where $f=1$ signifying a perfectly elastic material.

$$
G(t)=C_{0}+\sum_{i=1}^{2} C_{i} \exp \left(-t / \tau_{i}\right) .
$$

\subsection{Permeability}

Intrinsic permeability $(k)$ was calculated using a custom rig to simulate fluid flow through a porous medium as described by Darcy's law (Equation 3) [39]. Specimen dimensions were measured for thickness $(L)$ and cross sectional area $(A)$ then secured within the rig. 9 test samples were used with a diameter of $10 \mathrm{~mm}$ and an average thickness of $3.62 \pm 1.44 \mathrm{~mm}$. Pressure differences $(\Delta P)$ were measured with an applied flow rate of $0.1 \mathrm{~mL} / \mathrm{hr}$ and $0.05 \mathrm{~mL} / \mathrm{hr}(Q)$ of distilled water with a viscosity $(\mu)$ of $8.90 \times 10^{-4}$ Pa.s. Tests were allowed to equilibriate over 48 hours.

$$
Q=-\frac{k A \triangle P}{\mu L}
$$

\subsection{Cell Viability}

Cellular evaluations of gels and gel-infused scaffolds were carried out using NIH 3T3 murine fibroblasts. Gel concentrations of $2 \%$ and $3 \%$ were used to compare cell mobility throughout the biomaterials. 36 samples were cut to dimensions of $5 \mathrm{~mm} \times 5 \mathrm{~mm} \times 3 \mathrm{~mm}$ using a scalpel blade and placed in a 48 well culture plate. Cells were seeded on top of samples with a density of $5 \times 10^{4}$ and incubated in a humidified atmosphere of $37^{\circ} \mathrm{C}$ and $5 \% \mathrm{CO}_{2}$. Samples were cultured for 21 days in Dulbecco's Modified Eagles Medium (DMEM) supplemented with $10 \%$ fetal bovine serum and $1 \%$ Penicillin-Streptomycin, with the culture medium changed every other day.

An AlamarBlue test was carried out to assess cell proliferation by adding $10 \%$ AlamarBlue solution in DMEM and incubated for 4 hours at $37^{\circ} \mathrm{C}$. Absorbance was monitored using a fluorescence microplate reader (BMG Labtech Fluostar Optima Microplate reader; Ortenberg, Germany) at wavelengths of 544 and $590 \mathrm{~nm}$. Absorbance of solutions were proportional to the cell metabolism and population in each sample. Readings were taken at 7, 14 and 21 days after cell culture.

Cell numbers were quantified by generating a calibration curve from known cell numbers. Cells were seeded at densities of $1.5 \times 10^{4}, 5 \times 10^{4}, 8 \times 10^{4}, 2 \times 10^{5}$, $7 \times 10^{5}$, and $10 \times 10^{5}$ and incubated for 6 hours. AlamarBlue tests were carried out using the same procedure and graphed against absorbance readings.

Cell morphology was investigated after 14 days to examine cellular viability and proliferation. A Live/Dead assay was performed using calcein and ethidium homodimer-1 dyes which bind to cell DNA causing live and dead cells to fluoresce green or red respectively. Images of the cultured cells on gels and gelinfused scaffolds were obtained using an Olympus CKX41 inverted microscope (Olympus, Japan). 


\subsection{Statistical Methods}

Data were examined with independent variables of gel concentration and the inclusion of a polymer scaffold network. Analyses were carried out on IBM SPSS Statistics (Armonk, NY, USA).

\section{Results}

\subsection{Mechanical Testing}

Mechanical properties derived from the indentation of pure gels and IPN scaffolds can be seen in Figure 2. An analysis of covariance (ANCOVA) was performed on transformed data for instantaneous modulus and equilibrium modulus readings. Levene's test indicated unequal variances across instantaneous $(\mathrm{F}=4.88, \mathrm{p}<.001)$ and equilibrium $(\mathrm{F}=20.03, \mathrm{p}<.001)$ modulus groups, so data was transformed using logarithmic methods previously described [18]. Original viscoelastic values were used for statistical analysis.

Instantaneous moduli were observed to significantly increase as a function of gel concentration after controlling for scaffold inclusion $\left(\mathrm{F}_{1,65}=586, \mathrm{p}<\right.$ $\left..001, \eta_{\mathrm{p}}^{2}=.900\right)$. Similarly, greater gel concentrations across biomaterial types demonstrated a significant increase in equilibrium moduli $\left(\mathrm{F}_{1,65}=618, \mathrm{p}<.001\right.$, $\left.\eta_{p}^{2}=.905\right)$. However, viscoelastic ratios did not show significant differences as a result in gel concentrations $\left(\mathrm{F}_{1,65}=1.806, \mathrm{p}=.184, \eta_{\mathrm{p}}^{2}=.027\right)$. In contrast, viscoelastic ratios were found to significantly decrease with the addition of polymer networks after controlling for gel concentrations $\left(\mathrm{F}_{1,65}=4.101, \mathrm{p}=.047\right.$, $\left.\eta_{\mathrm{p}}^{2}=.059\right)$.

The presence of polymer networks exhibited significantly higher material stiffnesses compared to hydrogels alone in both instantaneous $\left(\mathrm{F}_{1,65}=926, \mathrm{p}\right.$ $\left.<.001, \eta_{\mathrm{p}}^{2}=.934\right)$ and equilibrium cases $\left(\mathrm{F}_{1,65}=960, \mathrm{p}<.001, \eta_{\mathrm{p}}^{2}=.937\right)$. The modulus values displayed by IPN scaffolds are comparable values with the native nucleus pulposus [32] and suggests the customisability of material stiffness whilst retaining the material elastic characteristics.

\subsection{Permeability}

An ANCOVA showed that gel-polymer composites displayed significantly greater permeabilities than gels alone $\left(\mathrm{F}_{1,6}=6.171, \mathrm{p}=.048, \eta_{\mathrm{p}}^{2}=.507\right)$, indicating the polymeric network provided a means for fluid movement throughout the material. Permeabilities of gel concentrations of $2 \%$ and $3 \%$ did not significantly differ after controlling for scaffold presence $\left(\mathrm{F}_{1,6}=0.002, \mathrm{p}=.964, \eta_{\mathrm{p}}^{2}<\right.$ $.001)$. The permeability increase with gel concentration in polymer-reinforced samples compared to decreased values in pure gels suggested incomplete infusion throughout the polymer scaffolds (Figure 3 ). Greater gel concentrations are associated with higher gelation temperatures and a less fluidic solution during infusion. The presence of encapsulated air may contribute to the large permeabilities within the pure and scaffold-reinforced gels although visualisation 

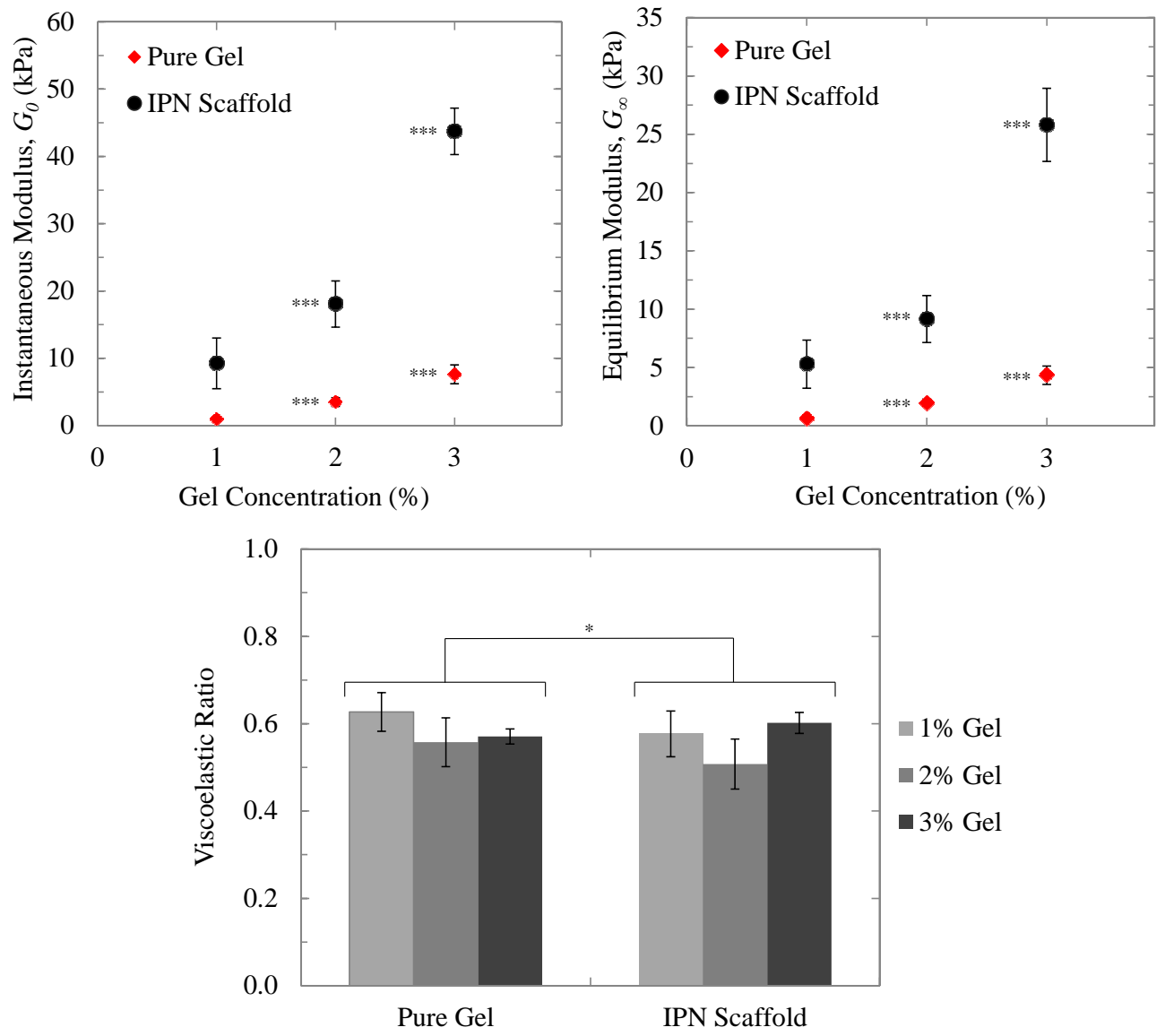

Figure 2: Instantaneous shear modulus $\left(G_{o}\right)$, equilibrium shear modulus $\left(G_{\infty}\right)$ and viscoelastic ratio $\left(f=G_{\infty} / G_{o}\right)$ of homogenous carrageenan gels and IPN composites plotted as a function of gel concentration as determined by spherical microindentation. Modulus values significantly increase with polymer network reinforcement and as a function of carrageenan content, while viscoelastic ratio remained relatively constant. Symbol signifies statistical significance where $* \mathrm{p}<0.05$ and ${ }^{* * *} \mathrm{p}<0.001$. 


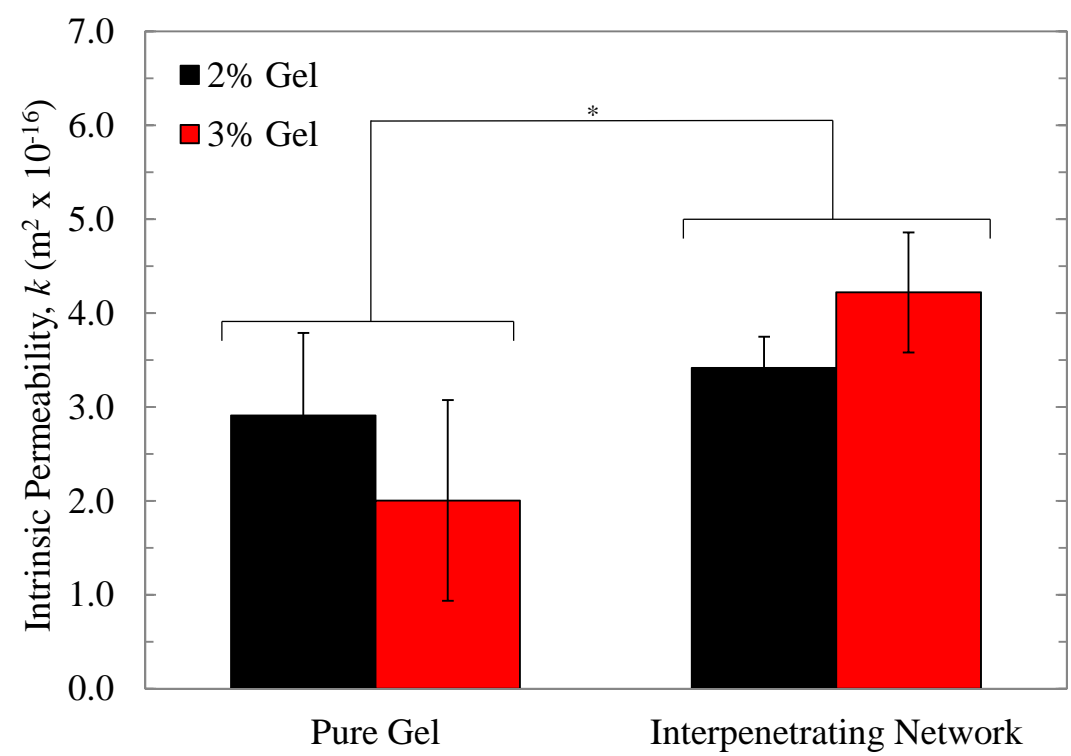

Figure 3: Intrinsic permeabilities $(k)$ of gels and gel-polymer composites with $2 \%$ and $3 \%$ concentrations determined using Darcy's Law. While 3\% gel concentrations indicated lower permeability in pure gels, the incorporation of polymer networks demonstrated a significantly inverse effect. Symbol signifies statistical significance where $* p<0.05$.

of these samples indicate that current protocols result in minimal trapped air within final biomaterials. Permeabilities of both pure and reinforced gels were significantly larger than recorded values for the native nucleus pulposus however limited studies were found for human values and may be highly influenced by testing protocols and the constitutive methods for data analysis [13, 4].

\subsection{Cellular Evaluation}

Cell populations were tested against gel concentration and the presence of polymer networks using an ANCOVA. Pure PCL scaffolds were considered as gel concentrations of $0 \%$. As exhibited in Table 1, cell populations were significantly greater in smaller gel concentrations compared to larger gel concentrations after 7 and 14 days $(\mathrm{p}<.001)$ however were not significant after 21 days $(\mathrm{p}=.629)$. Initial cell numbers after 7 days in gel-polymer composites and pure gels indicated fibroblastic preference to the presence of polymer networks however this was not deemed significant $(\mathrm{p}=.362)$. The support of PCL networks was more pronounced after two weeks and cell numbers of IPN scaffolds reached similar values as PCL scaffolds after 21 days. This suggested polymeric networks within gel-infused composites significantly enhanced cellular proliferation over time compared to gels alone. These results indicate a significant change in cell growth over time, suggesting gel degradation. 
Cell viability was visualised using a Live/Dead assay after 14 days as highlighted in Figure 5 to investigate cellular mobility in biomaterials. Green and red fluoresence indicate live and dead cells respectively. From the Live/Dead assay results, more live cells had spread across the pure PCL scaffold compared to the localised cell clusters along the outer periphery of the carrageenan gel. Cells did not proliferate within the hydrogel structure and primarily remained on superficial areas of the gel biomaterial, further highlighted by the absence of dead cells within the hydrogel construct. A rounded morphology was observed on carrageenan gels suggesting cells were poorly attached to the gel surface with the absence of cytoplasmic projections. Moderate cellular proliferation can be seen in IPN scaffolds, primarily along PCL scaffold struts suggesting polymeric networks provided a means for cellular attachment through the gels. This difference in cell proliferation and population may be attributed to the increased access to media and surface area within polymer network constructs.

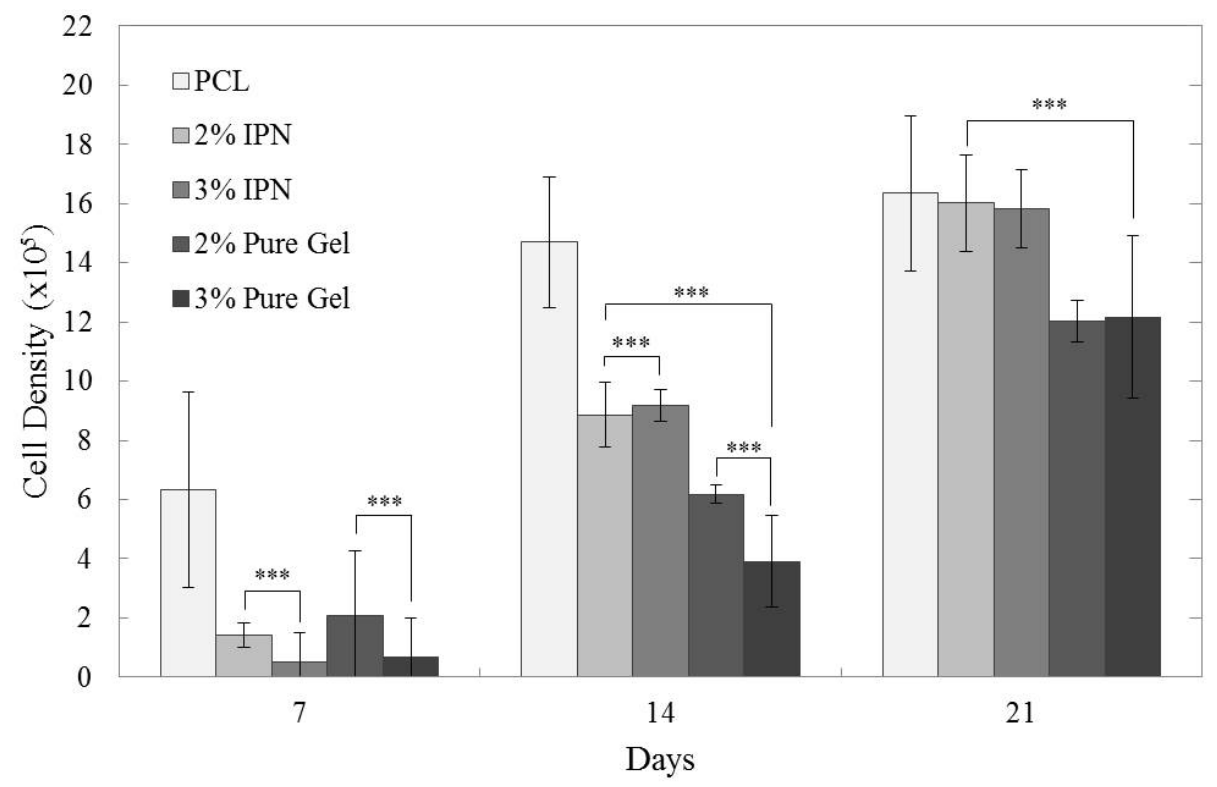

Figure 4: Total cell population measured using the AlamarBlue assay on carrageenan gels ( $2 \%$ GEL and $3 \%$ GEL), IPN scaffolds (2\% GEL-PCL and 3\% GEL-PCL) and PCL scaffolds over 21 days. Symbols signify statistical significance where ${ }^{* * *} \mathrm{p}<0.001$ as detailed in Table 1. 


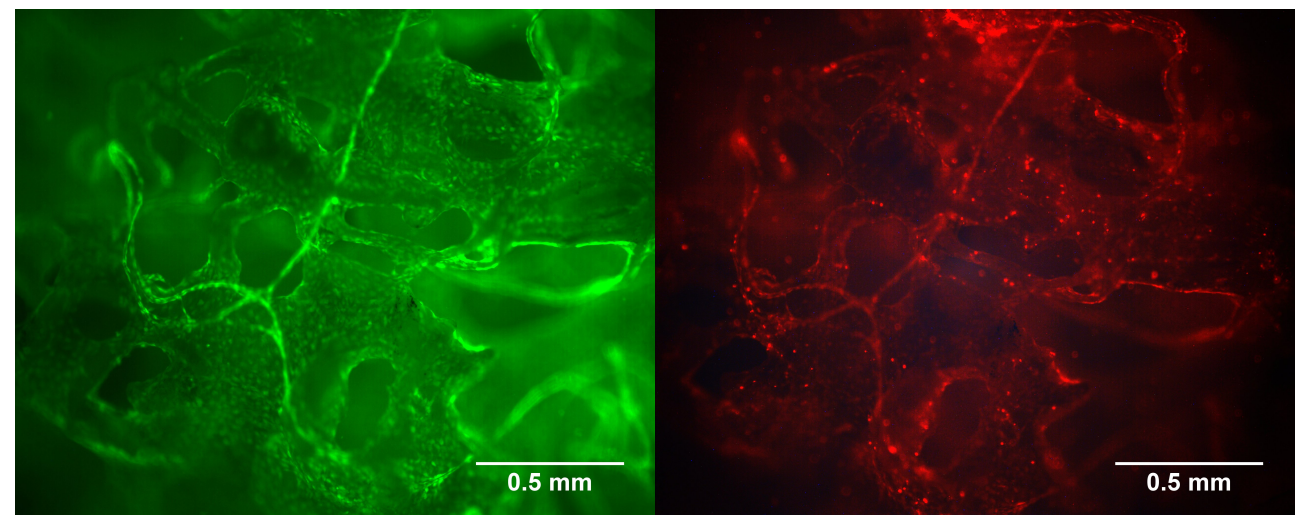

(a) PCL scaffold

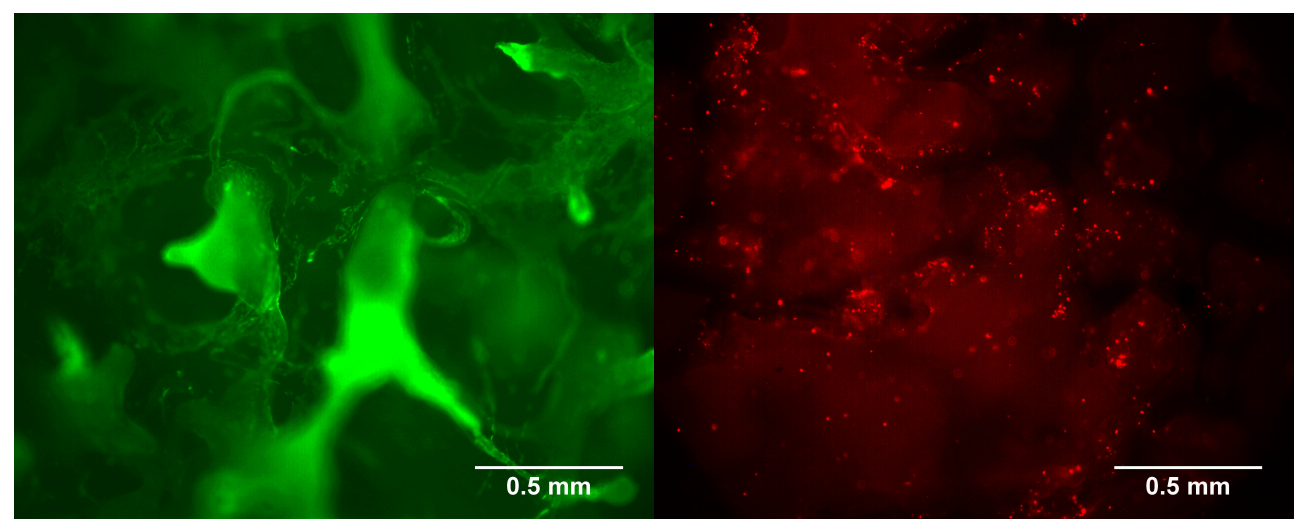

(b) Gel-infused scaffold

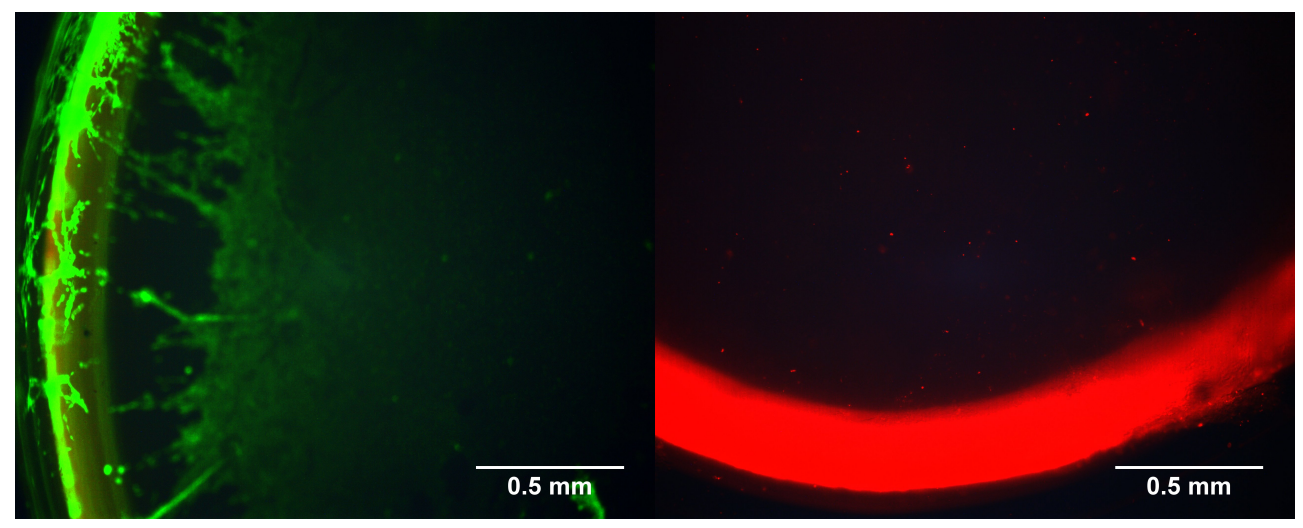

(c) Gel

Figure 5: NIH 3T3 murine fibroblasts visualised on PCL scaffolds, gel-polymer composites and carrageenan gels using an inverted fluorescence microscope under $4 \mathrm{x}$ magnification after 14 days of culture. Cellular proliferation was less pronounced in gel samples as cells were superficially located. Integration of polymer networks enhanced cell mobility with high and moderate levels observed in polymer scaffolds and gel-polymer composites respectively. 
Table 1: Analysis of covariance results on the effect of gel concentration and polymer network presence on cell population in gel and composite biomaterials after 7,14 and 21 days.

\begin{tabular}{|c|c|c|c|c|c|}
\hline Source & Days & df & $\mathrm{F}$ & $\mathrm{p}$ & $\eta^{2} p$ \\
\hline \multirow{3}{*}{$\begin{array}{l}\text { PCL Scaffold } \\
\text { Network Present }\end{array}$} & 7 & \multirow{3}{*}{1} & 0.862 & 0.362 & 0.032 \\
\hline & 14 & & 49.31 & $<0.001$ & 0.614 \\
\hline & 21 & & 23.622 & $<0.001$ & 0.417 \\
\hline \multirow{3}{*}{$\begin{array}{l}\text { Gel Concentration } \\
(\%)\end{array}$} & 7 & \multirow{3}{*}{1} & 36.427 & $<0.001$ & 0.584 \\
\hline & 14 & & 54.26 & $<0.001$ & 0.636 \\
\hline & 21 & & 0.237 & 0.629 & 0.007 \\
\hline
\end{tabular}

\section{Discussion}

In this study, IPN scaffolds were investigated through mechanical and cellular testing to assess its viability as a tissue engineered replacement for the nucleus pulposus. The integration of polymer networks within carrageenan gels increased moduli, permeabilities and promoted cellular growth and proliferation. These factors provide an exciting platform for further optimisation for the use of this composite material for disc regeneration.

The incompressibility of the composite was ensured using a gel phase within polymer networks, thereby increasing moduli whilst maintaining viscoelastic response. The mechanical properties of gel-infused scaffolds were comparable with value ranges of $5.4-44 \mathrm{kPa}$ previously obtained from the native nucleus pulposus when tested under unconfined pressure $[32,3]$. While measurements are significantly influenced by mechanical testing methods, the presented values may underestimate stiffnesses as the nucleus pulposus is known to withstand larger loads when confined by annular structures.

While this study focused on varied gel concentrations within polymer scaffolds, the mechanical behaviour of gel-infused polymers can similarly be altered by polymer structure and concentration. Studies by Strange et al. found the tensile modulus of alginate-infused PCL was related to the PCL fibres whereas indentation modulus was contingent on the alginate phase, whereby increasing gel concentrations reduces discrepancies between tensile and compressive moduli [35].

Hydraulic pressure is an integral function of the nucleus pulposus, supporting mechanical loads through the spine and providing resistance against compaction via viscous drag, electrostatic repulsion and osmotic swelling [11, 4]. Biomimetic materials therefore strive to replicate the low permeability properties of the nucleus pulposus to minimise alterations in mechanical response in vivo [6]. The permeability of both gels and IPN composites were orders of magnitudes higher than literature values of the human nucleus pulposus ranging between 0.67 $1.8 \times 10^{-18} \mathrm{~m}^{2}[25,9]$. Previous studies investigating gelatin-agar composites for nucleus pulposus tissue engineering attributed these elevated intrinsic permeabilities to the structural differences between gel and nucleus pulposus tissue, testing techniques and data analysis strategies [36]. 
As regenerative materials aim to facilitate tissue regrowth, a more permeable structure may be advantageous post-implantation to facilitate biomolecule exchange and movement. The intervertebral disc is one of the largest avascular parts of the body coupled with low cell density, therefore challenges remain to refine strategies for the tissues regeneration. Increased gel concentrations and the integration of polymer networks enhanced material stiffness and increased fluidic movement, striving toward a material that permits biomolecule movement within the scaffold as well as possessing high mechanical and swelling properties. While the exact parameters to engineer a nucleus pulposus are unknown, regeneration will likely require a higher biomolecule density than native tissue. The avascular nucleus pulposus lacks regenerative capacity and therefore a truly biomimetic construct may restrict the biomolecules needed to restore tissue, resemblant to the healing processes from an inflammatory response. An enhanced permeability may therefore be desirable to promote cellular growth and attachment, aiding tissue recovery under natural biomechanical cues [10, 34].

Findings from the preliminary cell evaluation in this study exhibited significantly higher proliferation in polymer-network biomaterials compared to gels. Structural elements provided a connective interface between the scaffolds and gels to facilitate cell proliferation. NIH 3T3s did not readily attach to gels as signified by the rounded morphology and superficial migration across gel surfaces. These results were also found by Mehrban et al suggesting cells were tightly bound within gel networks which hindered cellular migration, however proliferation was more pronounced in $\kappa$-carrageean compared to alginate [23]. This may indicate how cell populations in greater gel concentrations were initially significantly lower compared to lesser gel concentrations as the networks constricted cell mobility. Similar findings were demonstrated by Harris et al, who hypothesized that cell attachment was prevented due to poor cell traction across the material surfaces required to allow migration [8]. In contrast, carrageenan has been found to enhance cellular adhesion, morphology and proliferation of osteoblasts for composites including hydroxyapatite and chitosan for bone tissue engineering $[19,31]$.

While the interpenetrating network scaffolds supported NIH 3T3 proliferation, the fibroblastic nature of the cells evaluated are a limitation of this study. Nucleus pulposus cells are more chondrocyte in nature and therefore are likely to demonstrate different attachment and proliferation behaviours than fibroblasts. Studies by Popa et al. have shown $\kappa$-carrageenan to support the viability, proliferation and chondrogenic differentiation of encapsulated human adipose stem cells for cartilage regeneration [26]. Pronounced increases in stiffness and viscoelastic properties were also found in $\kappa$-carrageenan gel encapsulated adipose stem cells that were cultured in chondrogenic medium over time [28, 27]. This is in contrast to observed decreases in mechanical integrity of the $\kappa$-carrageenan gels during cell viability tests, consistent with fibroblastic studies with gels $[23,1]$. As nucleus pulposus cells display chondrocytic qualities, further investigation into cellular behaviour from the interplay between gel and polymer network would be recommended.

This interpenetrating composite combining PCL and carrageenan was devel- 
oped and investigated mechanically and biologically. Gel augmentation of the scaffold addressed the mechanical deficiencies often associated with hydrogels and demonstrated moduli proportional to the native nucleus pulposus. Measured permeabilities were notably higher than native values however this may facilitate tissue regeneration within the biomaterial structure post-implantation. Additionally, carrageenan gels readily supported cellular growth with the inclusion of polymer networks, enhancing cellular proliferation throughout the biomaterial. While the presented results are preliminary in nature, these findings open opportunities in tailoring this composite toward a suitable solution for nucleus pulposus regeneration.

\section{References}

[1] Basilia, B. A., Robles, A. P., Ledda, K. A., Dagbay, K. B., 2008. In-vitro and in-vivo screenings of electrospun polycaprolactone-carrageenan nanofibrous scaffolds for tissue engineering. In: Technical Proceedings of the 2008 NSTI Nanotechnology Conference and Trade Show, NSTI-Nanotech, Nanotechnology 2008. Vol. 2. pp. 306-309.

[2] Borges, A. C., Eyholzer, C., Duc, F., Bourban, P. E., Tingaut, P., Zimmermann, T., Pioletti, D. P., Manson, J. A. E., 2011. Nanofibrillated cellulose composite hydrogel for the replacement of the nucleus pulposus. Acta Biomaterialia 7 (9), 3412-3421.

[3] Cloyd, J. M., Malhotra, N. R., Weng, L., Chen, W., Mauck, R. L., Elliott, D. M., 2007. Material properties in unconfined compression of human nucleus pulposus, injectable hyaluronic acid-based hydrogels and tissue engineering scaffolds. European Spine Journal 16, 1892-1898.

[4] Cortes, D. H., Jacobs, N. T., DeLucca, J. F., Elliott, D. M., 2014. Elastic, permeability and swelling properties of human intervertebral disc tissues: A benchmark for tissue engineering.

[5] Garg, T., Singh, O., Arora, S., Murthy, R., jan 2012. Scaffold: a novel carrier for cell and drug delivery. Critical reviews in therapeutic drug carrier systems 29 (1), 1-63.

[6] Gloria, A., Causa, F., De Santis, R., Netti, P. A., Ambrosio, L., 2007. Dynamic-mechanical properties of a novel composite intervertebral disc prosthesis. Journal of Materials Science: Materials in Medicine 18 (11), $2159-2165$.

[7] Goins, M. L., Wimberley, D. W., Yuan, P. S., Fitzhenry, L. N., Vaccaro, A. R., 2005. Nucleus pulposus replacement: An emerging technology. Spine Journal 5 (6 SUPPL.), 317S-324S. 
[8] Harris, J. J., Smith, A. M., Campbell-Lynch, S., Shelton, R. M., 2008. Dramatic Changes in Bulk Deformation Behaviour of Gellan Gum on CrossLinking with Mixed Cations. In: Gums and Stabilisers for the Food Industry 14. The Royal Society of Chemistry, pp. 79-86.

[9] Heneghan, P., Riches, P. E., 2008. The strain-dependent osmotic pressure and stiffness of the bovine nucleus pulposus apportioned into ionic and non-ionic contributors. Journal of Biomechanics 41 (11), 2411-2416.

[10] Huang, Y.-c., Jill, P., Urban, G., Luk, K. D. K., 2014. Intervertebral disc regeneration: do nutrients lead the way? Nature Publishing Group 10 (9), $561-566$.

[11] Hunter, C., 2011. Fibrocartilage Tissue Engineering. In: Burdick, J., Mauck, R. (Eds.), Biomaterials for Tissue Engineering Applications SE - 13. Springer Vienna, pp. 363-387.

[12] Husson, J. L., Korge, A., Polard, J. L., Nydegger, T., Kneubühler, S., Mayer, H. M., 2003. A memory coiling spiral as nucleus pulposus prosthesis: Concept, specifications, bench testing, and first clinical results. Journal of Spinal Disorders and Techniques 16 (4), 405-411.

[13] Johannessen, W., Elliott, D. M., dec 2005. Effects of Degeneration on the Biphasic Material Properties of Human Nucleus Pulposus in Confined Compression. Spine 30 (24), E724-E729.

[14] Johnson, K. L., 1985. Contact Mechanics. Vol. 37. Cambridge University Press.

[15] Katz, J. N., 2006. Lumbar disc disorders and low-back pain: Socioeconomic factors and consequences. Journal of Bone and Joint Surgery - Series A 88 (SUPPL. 2), 21-24.

[16] Klara, P. M., Ray, C. D., jun 2002. Artificial Nucleus Replacement. Spine 27 (12), 1374-1377.

[17] Korge, A., Nydegger, T., Polard, J., Mayer, H., Husson, J., 2002. A spiral implant as nucleus prosthesis in the lumbar spine. European Spine Journal 11 (SUPPL. 2), S149-S153.

[18] Larson, M. G., 2008. Analysis of variance. Circulation 117 (1), 115-121.

[19] Liu, H., Cheng, J., Chen, F., Hou, F., Bai, D., Xi, P., Zeng, Z., 2014. Biomimetic and cell-mediated mineralization of hydroxyapatite by carrageenan functionalized graphene oxide. ACS Applied Materials and Interfaces 6 (5), 3132-3140.

[20] Marcolongo, M. S., Cannella, M., Massey, C. J., 2006. Chapter 10 - Nucleus Replacement of the Intervertebral Disc. Academic Press, Burlington, pp. 281-302. 
[21] Martino, A. D., Vaccaro, A. R., Lee, J. Y., Denaro, V., Lim, M. R., 2005. Nucleus Pulposus Replacement Basic Science and Indications for Clinical Use 30 (16), 16-22.

[22] Mattice, J. M., Lau, A. G., Oyen, M. L., Kent, R. W., 2006. Spherical indentation load-relaxation of soft biological tissues. Journal of Materials Research 21 (08), 2003-2010.

[23] Mehrban, N., Hunt, N., Smith, A. M., Grover, L. M., 2010. A comparative study of iota carrageenan, kappa carrageenan and alginate hydrogels as tissue engineering scaffolds. Gums and stabilisers for the food industry, 407-413.

[24] Mihaila, S. M., Gaharwar, A. K., Reis, R. L., Marques, A. P., Gomes, M. E., Khademhosseini, A., jun 2013. Photocrosslinkable kappa-carrageenan hydrogels for tissue engineering applications. Advanced healthcare materials 2 (6), 895-907.

[25] Périé, D., Iatridis, J. C., Demers, C. N., Goswami, T., Beaudoin, G., Mwale, F., Antoniou, J., jan 2006. Assessment of compressive modulus, hydraulic permeability and matrix content of trypsin-treated nucleus pulposus using quantitative MRI. Journal of biomechanics 39 (8), 1392-400.

[26] Popa, E. G., Caridade, S. G., Mano, J. F., Reis, R. L., Gomes, M. E., 2015. Chondrogenic potential of injectable k-carrageenan hydrogel with encapsulated adipose stem cells for cartilage tissue-engineering applications. Journal of Tissue Engineering and Regenerative Medicine 9 (5), 550-563.

[27] Popa, E. G., Caridade, S. G., Mano, J. F., Reis, R. L., Gomes, M. E., Caridade, G., Mano, J. F., Reis, R. L., Gomes, M. E., jan 2013. Chondrogenic potential of injectable k-carrageenan hydrogel with encapsulated adipose stem cells for cartilage tissue-engineering applications. Journal of Tissue Engineering and Regenerative Medicine, $\mathrm{n} / \mathrm{a}-\mathrm{n} / \mathrm{a}$.

[28] Popa, E. G., Carvalho, P. P., Dias, A. F., Santos, T. C., Santo, V. E., Marques, A. P., Viegas, C. A., Dias, I. R., Gomes, M. E., Reis, R. L., jan 2014. Evaluation of the in vitro and in vivo biocompatibility of carrageenanbased hydrogels. Journal of Biomedical Materials Research Part A, n/a$\mathrm{n} / \mathrm{a}$.

[29] Prajapati, V. D., Maheriya, P. M., Jani, G. K., Solanki, H. K., may 2014. Carrageenan: A natural seaweed polysaccharide and its applications. Carbohydrate Polymers 105, 97-112.

[30] Qiang, B., Greenleaf, J., Oyen, M., Zhang, X., 2011. Estimating material elasticity by spherical indentation load-relaxation tests on viscoelastic samples of finite thickness. IEEE Transactions on Ultrasonics, Ferroelectrics, and Frequency Control 58 (7), 1418-1429. 
[31] Santo, V. E., Frias, A. M., Carida, M., Cancedda, R., Gomes, M. E., Mano, J. F., Reis, R. L., 2009. Carrageenan-based hydrogels for the controlled delivery of PDGF-BB in bone tissue engineering applications. Biomacromolecules 10 (6), 1392-1401.

[32] Séguin, C. a., Grynpas, M. D., Pilliar, R. M., Waldman, S. D., Kandel, R. a., 2004. Tissue engineered nucleus pulposus tissue formed on a porous calcium polyphosphate substrate. Spine 29 (12), 1299-1306; discussion $1306-1307$.

[33] Sharifi, S., Bulstra, S. K., Grijpma, D. W., Kuijer, R., feb 2014. Treatment of the degenerated intervertebral disc; closure, repair and regeneration of the annulus fibrosus. Journal of Tissue Engineering and Regenerative Medicine, $\mathrm{n} / \mathrm{a}-\mathrm{n} / \mathrm{a}$.

[34] Sobol, E., Shekhter, A., Baskov, A., Baskov, V., Baum, O., Borchshenko, I., Golubev, V., Guller, A., Kolyshev, I., Omeltchenko, A., Sviridov, A., Zakharkina, O., Technologies, I., Sobol, E., Shekhter, A., Baskov, A., V., B., Baum, O., Borchshenko, I., Golubev, V., Guller, A., Kolyshev, I., Omeltchenko, A., Sviridov, A., Zakharkina, O., 2009. Regeneration of spine disc and joint cartilages under temporal and space modulated laser radiation. Progress in Biomedical Optics and Imaging - Proceedings of SPIE 7179, 71790B-71790B-7.

[35] Strange, D., Tonsomboon, K., Oyen, M. L., 2012. Electrospun FiberHydrogel Composites for Nucleus Pulposus Tissue Engineering. In: Materials Research Society. Vol. 1417. pp. 35-40.

[36] Strange, D. G. T., Oyen, M. L., jul 2012. Composite hydrogels for nucleus pulposus tissue engineering. Journal of the mechanical behavior of biomedical materials $11,16-26$.

[37] Strange, D. G. T., Tonsomboon, K., Oyen, M. L., 2014. Mechanical behaviour of electrospun fibre-reinforced hydrogels. Journal of Materials Science: Materials in Medicine 25 (3), 681-690.

[38] Thorvaldsson, A., Silva-Correia, J., Oliveira, J. M., Reis, R. L., Gatenholm, P., Walkenstrom, P., 2013. Development of nanofiber-reinforced hydrogel scaffolds for nucleus pulposus regeneration by a combination of electrospinning and spraying technique. Journal of Applied Polymer Science 128 (2), 1158-1163.

[39] Wang, H., 2000. Theory of Linear Poroelasticity with Applications to Geomechanics and Hydrogeology. Princeton series in geophysics. Princeton University Press.

[40] Xu, W., Ma, J., Jabbari, E., jun 2010. Material properties and osteogenic differentiation of marrow stromal cells on fiber-reinforced laminated hydrogel nanocomposites. Acta Biomaterialia 6 (6), 1992-2002. 